\title{
Hiperuricemia assintomática - tratar ou não tratar? - Uma revisão baseada na evidência
}

\author{
Asymptomatic hyperuricemia - to treat or not to treat? - An evidence based review \\ Hiperuricemia asintomática - ¿tratar o no tratar? - Una revisión basada en la evidencia \\ Helena Maria Ribeiro Fernandes ${ }^{a}$, Ana Catarina Andrade Peixoto ${ }^{a}$, Bruno Santos Maia ${ }^{b}$, Filipe Ribeiro Melo ${ }^{b}$, \\ Pedro Nuno Rego Mirandab
}

\section{Resumo}

Introdução: A avaliação dos níveis séricos de ácido úrico é realizada com frequência nos Cuidados de Saúde Primários, porém sem evidência científica que a justifique. A abordagem terapêutica subsequente constitui frequentemente um desafio clínico, particularmente no caso da hiperuricemia assintomática (HA). O objetivo desta revisão foi rever a evidência sobre a pertinência do tratamento da HA. Métodos: Pesquisa de normas de orientação clínica (NOC), revisões sistemáticas (RS), meta-análises (MA) e estudos originais (EO) no Medline e outros sítios de Medicina Baseada na Evidência, publicados desde abril de 2012 até abril de 2016, em inglês, espanhol e português. Termos $\mathrm{MeSH}$ : "hyperuricemia" e "asymptomatic conditions". Resultados: Cinco estudos cumpriam os critérios de inclusão: uma MA, três RS e um EO. A MA e o EO recomendam o tratamento da HA, para a prevenção da disfunção renal e para prevenção de eventos cardiovasculares (CV), respetivamente. Duas RS não recomendam o tratamento da HA e uma recomenda uma decisão individualizada para valores de uricemia acima de $9 \mathrm{mg} / \mathrm{dL}$, particularmente para a prevenção da gota. Conclusões: A evidência científica disponível é escassa, com limitações, e controversa no que diz respeito à instituição de tratamento farmacológico. O significado clínico da HA e sua relação causal com ocorrência de crises agudas de gota, disfunção renal e doença cardiovascular ainda são incertos. Não existe evidência científica que justifique o tratamento farmacológico da HA em doentes assintomáticos (SOR B). São, por isso, necessários mais estudos, metodologicamente robustos e orientados para o paciente.

\begin{abstract}
Introduction: Assessment of serum uric acid is frequently done in Primary Health Care, although not scientifically recommended. The subsequent therapeutic approach is often a clinical challenge, particularly in the case of asymptomatic hyperuricemia $(\mathrm{AH})$. The aim of this study was to review the evidence on AH treatment. Methods: A research was conducted on Medline and evidence-based medical sites for articles published between April 2012 and April 2016 in English, Spanish or Portuguese using the keywords "hyperuricemia" and "asymptomatic conditions". Results: Five articles met the inclusion criteria: one meta-analysis (MA), three systematic reviews (SR) and one original study (OS). MA and $\mathrm{OS}$ recommend treatment of $\mathrm{AH}$, for the prevention of renal dysfunction and for the prevention of cardiovascular events $(\mathrm{CV})$, respectively. Two SR do not recommend treatment of $\mathrm{AH}$ and one says that pharmacological treatment should be considered after an individual assessment of risk/benefit ratio, particularly in the prevention of gout in subjects with serum uric acid above $9 \mathrm{mg} / \mathrm{dL}$. Conclusion: Very limited scientific data are available on the pharmacologic treatment of $\mathrm{AH}$, with limitations and controversial results. The clinical significance of $\mathrm{AH}$ and its causal relationship with occurrence of acute attacks of gout, renal dysfunction and cardiovascular disease are still uncertain. There is no scientific evidence to support the pharmacological treatment of HA in asymptomatic patients (SORB). Further studies, that are methodologically robust and oriented to the patient are needed.
\end{abstract}

Como citar: Fernandes H, Peixoto AC, Maia BS, Melo F, Miranda P. Hiperuricemia assintomática - tratar ou não tratar? - Uma revisão baseada na evidência. Rev Bras Med Fam Comunidade. 2017;12(39):1-6. http://dx.doi.org/10.5712/rbmfc12(39)1396
Palavras-chave:

Hiperuricemia

Doenças Assintomáticas

Ácido Úrico

Atenção Primária à Saúde
Keywords:

Hyperuricemia

Asymptomatic Condition

Uric Acid

Primary Health Care
Fonte de financiamento: declaram não haver. Parecer CEP: não se aplica. Conflito de interesses: declaram não haver. Procedência e revisão por pares: revisado por pares. Recebido em: 20/09/2016. Aprovado em: 08/01/2017. 


\section{Resumen}

Introducción: La evaluación de los niveles de ácido úrico es una práctica frecuente en los Cuidados de Salud Primarios, pero no hay evidencia científica. El enfoque terapéutico posterior es frecuentemente un desafío clínico, particularmente en el caso de la hiperuricemia asintomática (HA). Objetivo: Revisar la evidencia sobre la relevancia del tratamiento de la HA. Métodos: Búsqueda de normas de orientación clínica, revisiones sistemáticas (RS), meta-análisis (MA) y estudios originales (EO) en la MEDLINE y otros lugares de Medicina Basada en la Evidencia, publicados desde abril/2012 hasta abril/2016, en inglés, español y portugués. Términos MeSH: "hyperuricemia" e "asymptomatic conditions". Resultados: Cinco estudios cumplían los criterios de inclusión: una MA, tres RS y un EO. La MA y el EO recomiendan el tratamiento de la HA, para la prevención de la disfunción renal y de problemas cardiovasculares (CV), respectivamente. Dos RS no recomiendan el tratamiento de la HA y una recomienda una decisión individualizada para valores de uricemia superiores a $9 \mathrm{mg} / \mathrm{dL}$, particularmente para la prevención de la gota. Conclusiones: La evidencia científica disponible es escasa, con limitaciones, y controversia, en lo que se refiere a la institución del tratamiento farmacológico. La importancia clínica de la HA y su relación causal con la ocurrencia de ataques agudos de gota, disfunción renal y las enfermedades cardiovasculares siguen siendo inciertas. No hay evidencia científica que justifique el tratamiento farmacológico de la HA en pacientes asintomáticos (SOR B). Son necesarios más estudios, metodológicamente robustos y orientados al paciente.
Palabras clave:

Hiperuricemia

Enfermedades Asintomáticas

Ácido Úrico

Atención Primaria de Salud

\section{Introdução}

O ácido úrico é o produto final do metabolismo das purinas. A hiperuricemia é definida por uma concentração sérica de ácido úrico igual ou superior a $6,8 \mathrm{mg} / \mathrm{dL}$, e é causada por uma produção excessiva de ácido úrico, uma diminuição da sua excreção ou por uma combinação de ambos. ${ }^{1}$

A avaliação dos níveis séricos de ácido úrico é realizada com frequência nos Cuidados de Saúde Primários, porém sem evidência científica que a justifique, sendo que a abordagem subsequente constitui frequentemente um desafio clínico, particularmente no caso da hiperuricemia assintomática (HA).

A incidência da HA tem aumentado nas últimas décadas, com prevalência estimada de cerca de $20 \%$ na população adulta. ${ }^{2} \mathrm{~A}$ HA é o principal fator de risco para a ocorrência de artrite gotosa, ${ }^{3,4}$ cerca de cinco vezes mais comum nos homens do que nas mulheres. No entanto, a maioria dos doentes com HA não irá desenvolver gota, com uma incidência anual estimada de cerca de $5 \%$ em indivíduos com níveis séricos superiores a $9 \mathrm{mg} / \mathrm{dL}^{5}$

Existe evidência de que a HA poderá aumentar o risco de desenvolvimento de doença renal, ${ }^{3-6}$ pela eventual deposição de cristais de urato nos ductos coletores e resposta inflamatória associada, bem como poderá ser um fator de risco cardiovascular (CV) independente, ${ }^{7}$ pela possível disfunção endotelial que condiciona. ${ }^{1,3,4}$ No entanto, a relação causal entre a HA e estas condições clínicas continua controversa. ${ }^{8}$

O tratamento da hiperuricemia pode englobar medidas não farmacológicas, pela alteração de estilos de vida, e tratamento farmacológico, com uso de hipouricemiantes, como o alopurinol, um inibidor da xantina oxídase. Este associa-se, com alguma frequência, ao desenvolvimento de reações cutâneas de hipersensibilidade, além de outros efeitos laterais menos frequentes, embora não desprezíveis, e deve ser utilizado com precaução nos doentes com insuficiência renal. ${ }^{9}$

O tratamento farmacológico da HA não é consensual, pela incerteza de que a diminuição dos níveis sérios de ácido úrico possa reduzir o risco de ocorrência de artrite gotosa, doença renal e eventos CV. O objetivo desta revisão consistiu em rever a evidência sobre a pertinência do tratamento da HA. 


\section{Métodos}

Foi realizada uma pesquisa bibliográfica de normas de orientação clínica (NOC), revisões sistemáticas (RS), meta-análises (MA) e estudos originais (EO) em 12 de abril de 2016, com os termos MeSH e operador booleano: "hyperuricemia" AND "asymptomatic conditions".

As bases de dados pesquisadas incluem: National Guideline Clearinghouse, Guideline Finder, Canadian Medical Association, The Cochrane Database, DARE, Bandolier, Medline/PubMed e Índex de Revistas Médicas Portuguesas.

Incluíram-se os artigos publicados de abril de 2012 até abril de 2016, em língua portuguesa, inglesa ou espanhola que respeitassem os critérios de elegibilidade a seguir especificados:

- População: doentes adultos, sem doença oncológica ativa nem doenças cardiovascular ou renal estabelecidas;

- Intervenção: tratamento não farmacológico ou farmacológico - hipouricemiante (alopurinol; outros hipouricemiantes - rasburicase, benzbromarona);

- Comparação: sem tratamento ou placebo;

- Outcome (orientado para o doente): prevenção de gota, doença renal ou eventos CV.

Definiram-se como critérios de exclusão:

- Presença de doentes portadores de hiperuricemia iatrogênica ou de causa congênita;

- Não utilização de qualquer tratamento na redução da HA;

- Estudos nos quais o grupo controle não padece de HA.

Para avaliação dos níveis de evidência (NE) e atribuição de forças de recomendação (FR), foi usada a escala Strenght of Recommendation Taxonomy (SORT), da American Family Physician. ${ }^{10}$

\section{Resultados}

Foram encontrados 99 artigos, tendo-se excluído as publicações repetidas, os artigos cujo tema não se relacionava com o objetivo do trabalho e os artigos que apresentavam critérios de exclusão. Cinco artigos cumpriam os critérios de inclusão: uma MA, três RS e um EO (Modelo de Markov), que foram revistos na sua totalidade.

Na MA incluída, Wang et al., ${ }^{6}$ foram analisados 11 Ensaios Clínicos Aleatorizados (ECA), que comparavam o tratamento hipouricemiante (alopurinol ou outros - rasburicase ou benzbromarona-) com o placebo, e tinham como outcome principal a prevenção da disfunção renal. Segundo esta MA, haverá indicação para o tratamento da HA. Dos estudos incluídos, um tinha dupla ocultação, dois tinham a ocultação apenas dos participantes, e os oito restantes não faziam referência à ocultação. Os autores consideram que serão ainda necessários mais ensaios clínicos randomizados e controlados com amostras maiores e com tempos de estudo superiores. (NE 2). 
Uma das RS, Graf et al., ${ }^{11}$ procurou analisar ECA que comparassem o tratamento hipouricemiante (com alopurinol) com o placebo, tendo como outcome principal a prevenção de doença renal, gota, e eventos CV. Nos três ECAs incluídos na revisão, não foram encontradas diferenças estatisticamente significativas no que diz respeito à taxa de filtração glomerular entre o grupo tratado e o grupo controle. Os ensaios não avaliavam a prevenção da gota nem de eventos CV. Segundo esta RS, não há evidência para o tratamento da HA no sentido de prevenir disfunção renal. Os três ECA foram de curta duração, compostos por um número reduzido de participantes e com risco significativo de viés (NE 2).

Numa outra RS incluída, Vinik et al., ${ }^{4}$ foram analisados dois ECA, que também comparavam o tratamento hipouricemiante (com alopurinol) com o placebo, tendo como outcome principal a prevenção de gota, doença renal e eventos CV. Não se encontraram diferenças estatisticamente significativas na função renal entre os pacientes tratados e não tratados. De acordo com esta RS, não há evidência para o para o tratamento da HA no sentido de prevenir gota, disfunção renal ou eventos CV. Os 2 ECA apresentavam como limitações uma ausência de dupla ocultação, o seu tempo limitado (12 meses) e o fato de serem compostos por um número reduzido de participantes (NE 2).

Uma das RS, Araújo et al., ${ }^{12}$ analisou os três ECA incluídos na RS de Graf et al., ${ }^{11}$ apresentando como conclusão não haver evidência para o tratamento da HA para a prevenção de disfunção renal (NE 2). No entanto, de acordo com esta RS, poderá haver evidência para o tratamento da HA, sendo que a decisão deverá ser individualizada, em doentes com valores de uricemia iguais ou superiores a $9 \mathrm{mg} / \mathrm{dL}$, e com o objetivo de prevenção da gota - recomendação baseada nos estudos observacionais de grande dimensão Framingham (1967) e Normative Aging (1987), que demonstraram um risco aumentado de desenvolvimento de gota para valores de uricemia $\geq 9 \mathrm{mg} / \mathrm{dL})(\mathrm{NE} 2)$.

O Estudo Original incluído, Akkineni et al., ${ }^{1}$ consistiu numa coorte hipotética, usando o Modelo de Markov, de indivíduos de 50 anos de idade, comparando o tratamento da hiperuricemia em todos os indivíduos (assintomáticos e sintomáticos), por oposição ao tratamento apenas dos indivíduos sintomáticos (com gota ou nefrolitíase). O outcome consistia na prevenção de eventos CV. Segundo este estudo, haverá redução da incidência e da mortalidade por eventos $\mathrm{CV}$, por meio do tratamento da HA com alopurinol, se valores de uricemia superiores a $7 \mathrm{mg} / \mathrm{dL}$, nos doentes do gênero masculino, e superiores a $5 \mathrm{mg} / \mathrm{dL}$, em doentes do gênero feminino. À medida que a população envelhece, o tratamento da HA tornar-se-á menos eficaz na prevenção de eventos CV.

Este estudo consiste no primeiro modelo de investigação clínica que compara estratégias de tratamento da HA na prevenção da morbimortalidade CV. Uma vez que as estimativas usadas no estudo são baseadas em dados criados/formulados pelo cruzamento da evidência disponível em estudos observacionais, não podem ser assumidas relações de causalidade. Os autores reconhecem que este estudo não deve ser utilizado como uma norma de orientação clínica (NE 2).

\section{Discussão}

A evidência científica disponível não permite assumir uma relação causal entre a presença de $\mathrm{HA} e$ condições clínicas, nomeadamente do foro cardiovascular e renal e artrite gotosa. 
No que diz respeito ao tratamento farmacológico da HA, a evidência que suporta ou refuta a sua instituição é escassa e com limitações, composta por estudos de tamanho amostral e tempos de seguimento reduzidos. Além disso, os resultados nem sempre fornecem respostas relativas a todos os outcomes previstos nos desenho iniciais dos estudos, sendo que em cada estudo são avaliados outcomes diferentes, o que dificulta a homogeneização dos resultados e conclusões.

O EO referido no presente estudo, sendo uma coorte hipotética, poderá ser utilizado como uma base para ensaios clínicos orientados para o doente e metodologicamente mais robustos, que são, indubitavelmente, necessários nesta temática.

Não existe evidência científica que justifique o tratamento farmacológico da HA em doentes assintomáticos (SOR B). Cabe ao médico de família, na posição privilegiada que assume na promoção da saúde e prevenção da doença dos indivíduos adultos saudáveis, efetuar uma prática clínica que tenha sempre como base uma evidência científica atualizada.

\section{Referências}

1. Akkineni R, Tapp S, Tosteson AN, Lee A, Miller KL, Choi HK, et al. Treatment of asymptomatic hyperuricemia and prevention of vascular disease: a decision analytic approach. J Rheumatol. 2014;41(4):739-48. DOI: http://dx.doi.org/10.3899/ jrheum.121231

2. Zhu Y, Pandya BJ, Choi HK. Prevalence of gout and hyperuricemia in the US general population: the National Health and Nutrition Examination Survey 2007-2008. Arthritis Rheum. 2011;63(10):3136-41. DOI: http://dx.doi.org/10.1002/art.30520

3. Viazzi F, Leoncini G, Pontremoli R. Cardiovascular and renal effects of hyperuricaemia and gout. Reumatismo. 2012;63(4):253-62. DOI: http://dx.doi.org/10.4081/reumatismo.2011.253

4. Vinik O, Wechalekar MD, Falzon L, Buchbinder R, van der Heijde DM, Bombardier C. Treatment of asymptomatic hyperuricemia for the prevention of gouty arthritis, renal disease, and cardiovascular events: a systematic literature review. J Rheumatol Suppl. 2014;92:70-4. DOI: http://dx.doi.org/10.3899/jrheum.140465

5. Emmerson BT. The management of gout. N Engl J Med. 1996;334(7):445-51. DOI: http://dx.doi.org/10.1056/ NEJM199602153340707

6. Wang $\mathrm{H}$, WeiY, Kong X, Xu D. Effects of urate-lowering therapy in hyperuricemia on slowing the progression of renal function: a meta-analysis. J Ren Nutr. 2013;23(5):389-96. DOI: http://dx.doi.org/10.1053/j.jrn.2012.08.005

7. Kim SY, Guevara JP, Kim KM, Choi HK, Heitjan DF, Albert DA. Hyperuricemia and coronary heart disease: a systematic review and meta-analysis. Arthritis Care Res (Hoboken). 2010;62(2):170-80. DOI: http://dx.doi.org/10.1002/acr.20065

8. Zechman E. What to Do--or Not--About Asymptomatic Hyperuricemia. [citado 17 Jan 2017]. Disponível em: http://www. medpagetoday.com/resource-center/Gout/Asymptomatic-Hyperuricemia/a/49781

9. Macías N, Goicoechea M, de Vinuesa MS, Verdalles U, Luño J. Urate reduction and renal preservation: what is the evidence? Curr Rheumatol Rep. 2013;15(12):386. DOI: http://dx.doi.org/10.1007/s11926-013-0386-3

10. Ebell MH, Siwek J, Weiss BD, Woolf SH, Susman J, Ewigman B, et al. Strength of recommendation taxonomy (SORT): a patient-centered approach to grading evidence in the medical literature. Am Fam Physician. 2004;69(3):548-56. DOI: http://dx.doi.org/10.3122/jabfm.17.1.59

11. Graf SW, Whittle SL, Wechalekar MD, Moi JH, Barrett C, Hill CL, et al. Australian and New Zealand recommendations for the diagnosis and management of gout: integrating systematic literature review and expert opinion in the $3 e$ Initiative. Int J Rheum Dis. 2015;18(3):341-51. DOI: http://dx.doi.org/10.1111/1756-185X.12557

12. Araújo F, Cordeiro I, Teixeira F, Rovisco J, Ramiro S, Mourão AF, et al. Portuguese recommendations for the diagnosis and management of gout. Acta Reumatol Port. 2014;39(2):158-71. 
a Unidade de Saúde Familiar do Mar, Agrupamento dos Centros de Saúde Grande Porto IV. Póvoa de Varzim/Vila do Conde, Portugal. helenafernandes25@gmail.com (Autora correspondente); catarinapeixoto@usfdomar.com

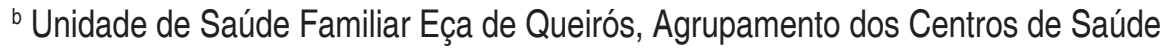
Grande Porto IV. Póvoa de Varzim/Vila do Conde, Portugal. bsmaia@msn.com; filipemelo1@gmail.com; pedronuno_miranda@hotmail.com 